\title{
Feasibility of enhanced recovery protocol in minimally invasive Mckeown esophagectomy
}

\author{
Yuichiro Tanishima ${ }^{1}$ - Katsunori Nishikawa ${ }^{1} \cdot$ Masami Yuda $^{1} \cdot$ Yoshitaka Ishikawa $^{1} \cdot$ Keita Takahashi $^{1}$. \\ Yujiro Tanaka ${ }^{1} \cdot$ Akira Matsumoto $^{1} \cdot$ Fumiaki Yano $^{1} \cdot$ Norio Mitsumori $^{1} \cdot$ Toru Ikegami $^{1}$
}

Received: 22 September 2020 / Accepted: 5 February 2021 / Published online: 18 February 2021

(c) The Japan Esophageal Society 2021

\begin{abstract}
Background Implementation of enhanced recovery after surgery has generally been applied to gastrointestinal surgeries; however, few studies have investigated minimally invasive McKeown esophagectomy. In this study, we aimed to evaluate the safety and feasibility of an enhanced recovery protocol after minimally invasive McKeown esophagectomy.

Methods Data were collected between January 2015 and April 2020 for patients who underwent esophagectomy. Of these patients, those who underwent minimally invasive McKeown esophagectomy was selected for the investigation. Perioperative outcomes and nutritional index were compared using propensity score matching between the conventional group and the enhanced recovery group.

Results A total of 119 patients were enrolled in this study. Of these, 73 and 46 were treated with conventional and enhanced recovery protocol, respectively. Forty-two pairs were matched in two groups. The enhanced recovery group showed a lower rate of pulmonary complications $(9.5 \%$ vs. $28.5 \%, p=0.0235)$, abdominal dysfunctions $(16.7 \%$ vs. $42.9 \%, p=0.0078)$, and shorter hospital stay as compared with the conventional group (17.5 days vs. 23 days, $p=0.0034)$. The loss of body weight (6.3\% vs. $7.7 \%, p=0.0065)$ and body mass index (5.6\% vs. $8.1 \%, p=0.0017)$ were significantly lower in the enhanced recovery group than in the conventional group. In contrast, nutritional biochemistry data did not differ significantly between the two groups.

Conclusions This study shows that the promotion of an enhanced recovery protocol in minimally invasive McKeown esophagectomy maintains nutritional status without increasing postoperative complications.
\end{abstract}

Keywords Enhanced recovery after surgery $\cdot$ Esophageal neoplasms $\cdot$ Esophagectomy $\cdot$ Postoperative complications ·

Nutritional status

\section{Introduction}

Esophageal cancer is the sixth most common cancer, with more than 400,000 deaths worldwide. Currently, esophagectomy is considered the best curative modality for esophageal cancer, with a 5-year survival rate of $55.6 \%[1,2]$. However, surgical damage and postoperative complications associated with esophagectomy are more severe than those associated with other digestive system surgeries. From a Japanese nationwide review, the postoperative outcomes

Yuichiro Tanishima

tanishima@jikei.ac.jp

1 Division of Gastrointestinal Surgery, Department of Surgery, The Jikei University School of Medicine, 3-25-8, Nishi-Shimbashi, Minato-ku, Tokyo 105-8461, Japan after esophagectomy are still unsatisfactory, with overall morbidity of $59 \%$, length of postoperative hospital stay of 41 days, and 30-day mortality of $2.4 \%$ [3]. Nutritional management and prevention of postoperative complications are two important factors that promote rapid recovery for esophageal cancer surgery.

A transition from open to endoscopic surgery has recently been applied to many types of surgery [4]. Combined thoracoscopy and laparoscopy for patients with esophageal malignancy have been used as minimally invasive esophagectomy (MIE). The indications for MIE have expanded with clinical evidence regarding oncological safety and advantages in postoperative management [5, 6]. In particular, an enhanced recovery after surgery (ERAS) protocol has become standard perioperative care to minimize postoperative complications and accelerate recovery [7]. On the other hand, the timing of 
extubation and diet rehabilitation after minimally invasive McKeown esophagectomy (MIMcE) remains controversial $[8,9]$.

In April 2018, we introduced an enhanced recovery protocol for MIMcE with the aim to improve the outcomes after MIMcE. Therefore, we aimed to examine the safety and feasibility of ERAS for MIMcE in the current study.

\section{Patients and methods}

\section{Patients}

A total of 137 patients with esophageal cancer who underwent MIMcE at the Department of Surgery, Jikei University Hospital between January 2015 and April 2020, were enrolled. In total, 137 patients underwent thoracoscopic esophagectomy. After excluding 18 patients who were not eligible for simultaneous reconstruction, laparoscopic surgery for gastric conduit, and cervical anastomosis, the remaining 119 patients were classified into two protocols. Of these, 73 patients were treated with the conventional protocol, and 46 patients were treated with a new protocol. The patients were matched in 42 pairs for clinical and demographic variables as follows: age; sex; body mass index (BMI); American Society of Anesthesiologists physical status (ASA-PS); and clinical stage using propensity scores, and divided into $\mathrm{C}$ and ER groups (Fig. 1).

Clinical TNM staging was diagnosed according to the TNM classification of malignant tumors (8th edition) before treatment with computed tomography (CT) and esophagogastroduodenoscopy (EGD). Patients with clinical stage IB-IVA disease were treated with preoperative chemotherapy.

This study was approved by the Institutional Review Board of the Jikei University Hospital (Approval number,

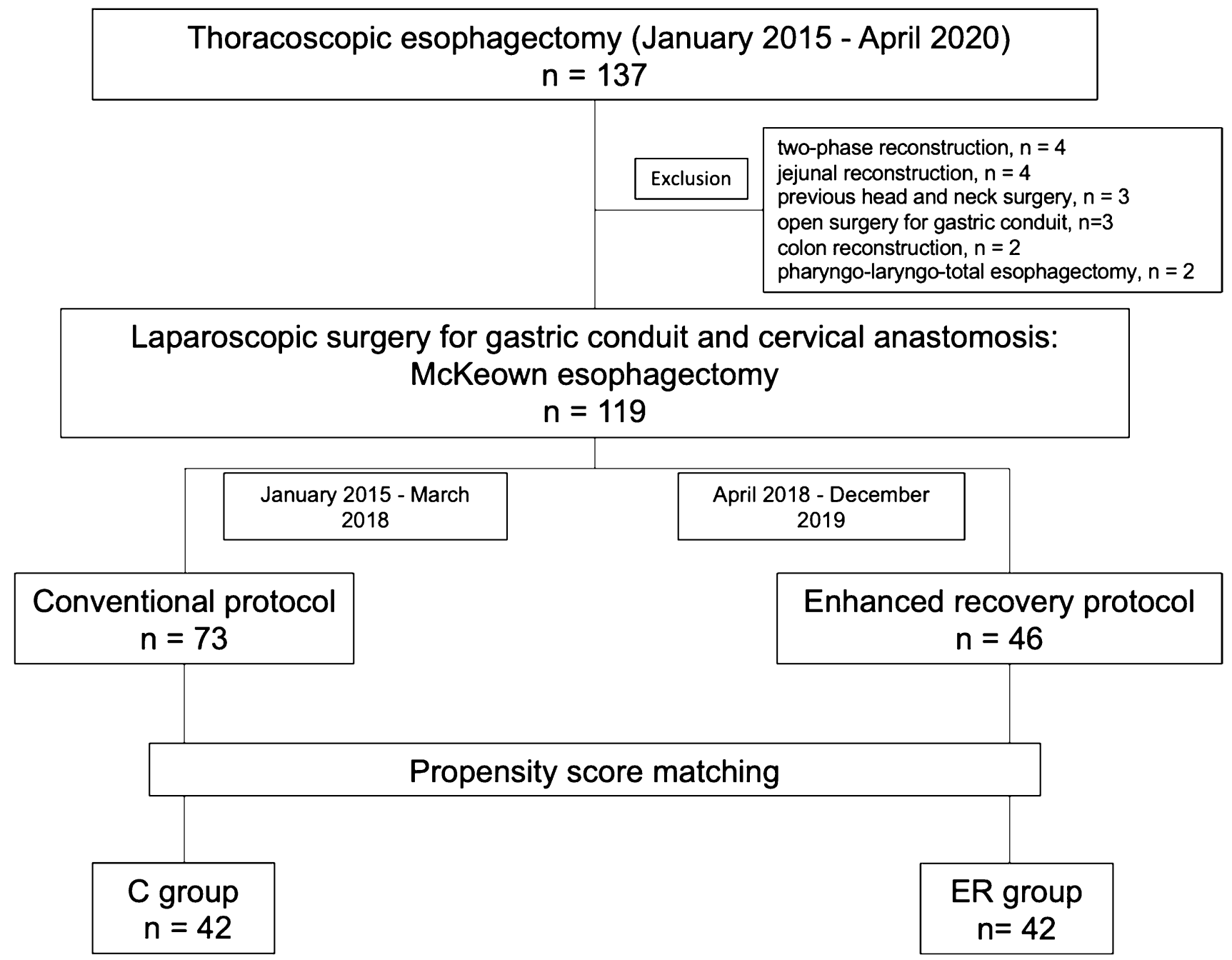

Fig. 1 Patients' tree. Patients in this study were matched for demographic variables by propensity scores, in the matched two-group consisting of 42 patients each 
28-054), and written informed consent was obtained from all patients.

\section{Surgical procedure}

The patients underwent thoracoscopic esophagectomy with two- or three-field lymphadenectomy according to the treatment guidelines in Japan [10]. Lymphadenectomy along the recurrent laryngeal nerves was securely performed with an intraoperative nerve integrated monitoring system to prevent nerve injury. After the thoracic procedure, gastric mobilization was performed laparoscopically. The gastric conduit was constructed extracorporeally and pulled up via the posteriormediastinal or retro-sternal route. An end-to-end anastomosis was created in the cervix (McKeown esophagectomy). Feeding button-type jejunostomy was routinely performed for adequate postoperative enteral nutrition (EN).

\section{Perioperative care}

\section{Conventional protocol}

Table 1 shows the conventional protocol from January 2015 to March 2018. Respiratory rehabilitation using the device was initiated before admission. Mechanical bowel preparation was routinely used with polyethylene glycol the day before surgery, and clear fluid was allowed until $10 \mathrm{~h}$ before surgery. At the end of the operation, two cervical drains and a single thoracic drain were placed, and a nasogastric (NG) tube was inserted for decompression of the gastric conduit. Extubation followed by a vocalization assessment with an anesthesiologist and removal of the NG tube were performed on a postoperative day (POD) 1 . EN was commenced from jejunostomy, increasing the volume gradually to $1680 \mathrm{kcal} /$ day by POD 7. Postoperative physiotherapy was started after transfer to the surgical ward from the intensive care unit (ICU) on POD 2. Cervical drains and a thoracic drain were generally removed on POD 3 and POD 7, respectively. Fasting was performed until POD 6. After a swallowing assessment by an otolaryngologist on POD 7, oral intake was resumed with an ice cube, followed by a soft diet on POD 8. Discharge was scheduled on POD 17 for a patient with a planned postoperative course.

\section{Enhanced recovery protocol}

The enhanced recovery protocol, in use since April 2018, is shown in Table 2. Respiratory rehabilitation was the same as in conventional protocol. Unlike the conventional protocol, bowel preparation was not required, and instead of clear fluid a solid meal was allowed before surgery. Extubation followed by vocal cord assessment and removal of the NG tube were performed immediately in the operating room.
Bedside physical rehabilitation was started in the ICU on POD 1. EN was increased in volume up to $960 \mathrm{kcal} / \mathrm{day}$ by POD 4. According to the ERAS concept, ice cube intake resumed on POD 2, and a soft fractionated diet was started on POD 4 after swallowing assessment. Discharge was generally scheduled on POD 12.

\section{Outcomes}

The following variables in the matched groups consisting of 42 patient pairs were compared: (1) postoperative clinical course; (2) postoperative complication; and a (3) nutritional assessment.

\section{Evaluation of the postoperative clinical course}

The following parameters were assessed: use of catecholamine, time to extubation, duration of ICU stay, time to ambulation, time to oral feeding, time to defecation/flatus, inflammatory response, and length of hospital stay.

\section{Complications}

Postoperative morbidity was analyzed according to the Clavien-Dindo classification (CD). Regarding the diagnosis of vocal cord palsy, each patient was routinely referred to an anesthesiologist at the time of extubation. Otolaryngologist conducted a swallowing assessment and examined hoarseness, pooling of saliva, and clearance to the esophagus with video endoscopy. We defined a pulmonary complication as the presence of clinical signs confirmed by chest X-ray or CT within 2 weeks postoperatively. Diagnosis of anastomotic leakage was based on the nature of the neck as well as CT and EGD. Chylothorax was diagnosed by either a change in milky white color of thoracic drainage, regardless of output, or confirmation of chylomicrons in the fluid with high-volume discharge.

\section{Nutritional assessment}

Maximum dosage of EN and total calorie intake were compared between two groups. Abdominal dysfunction related to EN was defined as gastro-intestinal complaints such as diarrhea and abdominal distention; the amount of tube feeding was reduced or temporarily suspended until the complaints resolved. We evaluated physical examination and biochemical nutritional markers before surgery and 1 month after esophagectomy. The biochemical nutritional markers included serum albumin (Alb), cholinesterase (ChE), and serum transthyretin (TTR). 


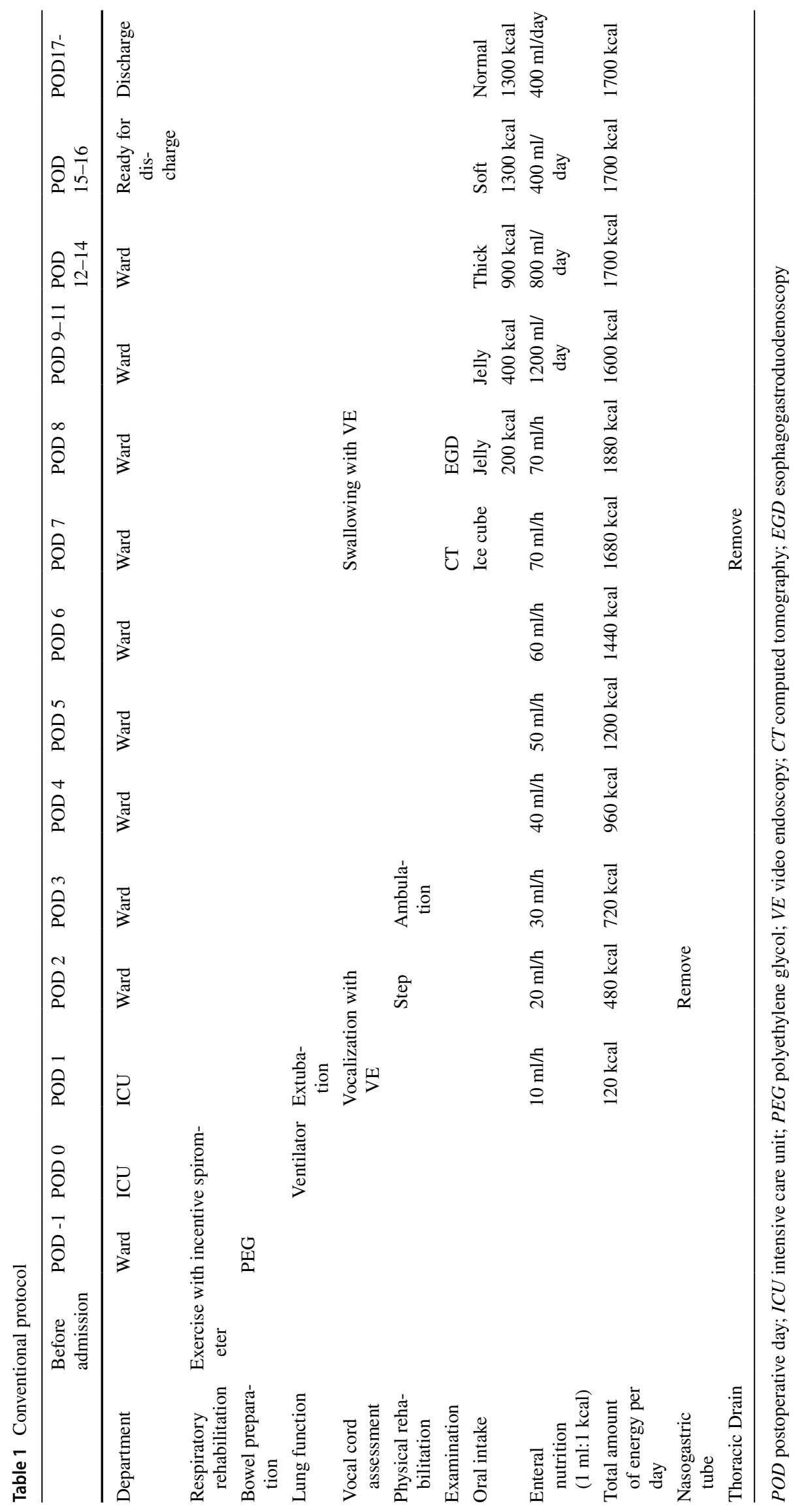




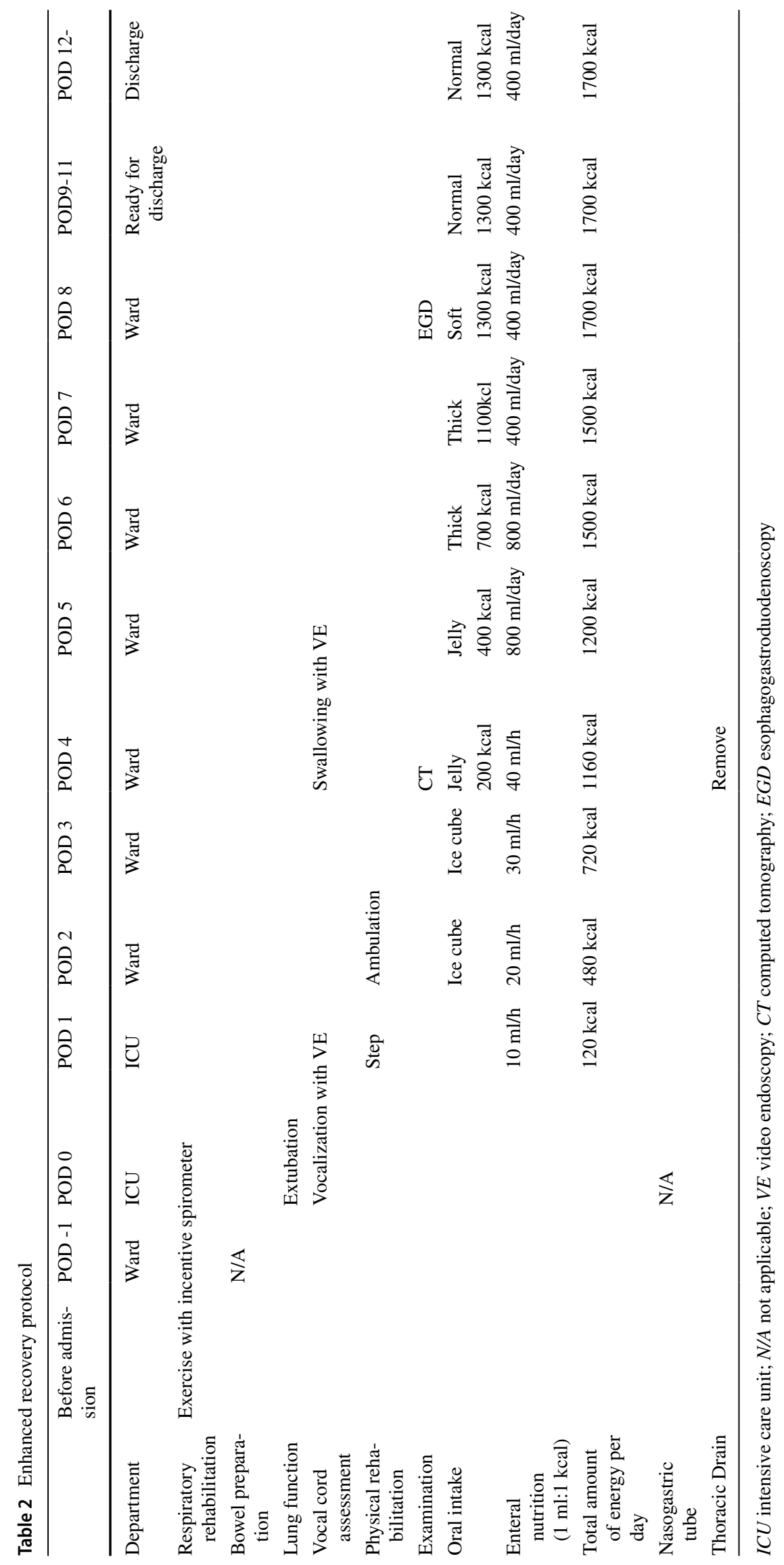




\section{Statistical analysis}

All quantitative data are expressed as median or mean \pm standard deviation. A propensity score was calculated for each participant using a logistic regression model to adjust for the following five covariates: age, sex, BMI, ASAPS, and clinical stage. Differences between the two groups were compared using the Student $t$ test, Wilcoxon rank-sum test, or Chi squared test. JMP version 14 (SAS Institute Inc., Cary, NC, USA) was used for statistical analysis. Statistical significance was considered at $p<0.05$.

\section{Results}

Table 3 shows demographic and clinical characteristics of the two protocols before and after matching. The patients in the current study were predominantly male, ASA-PS2 classification, and had squamous cell carcinoma. There were no significant differences in all covariates before and after matching.
Table 4 shows perioperative outcomes of the two protocols before and after matching. The two groups had similar intraoperative outcomes such as operative time, blood loss, fluid balance, and use of catecholamine. Time to extubation was shorter in ER group than in $\mathrm{C}$ group (0 days vs. 1 days, $p<0.0001)$. Postoperative use of catecholamine was lower in ER group than in C group ( $16.7 \%$ vs. $92.8 \%, p<0.0001)$. Length of ICU stay was similar in both the groups. Time to the first ambulation was shorter in ER group than in C group ( 2 days vs. 3 days, $p<0.0001$ ). Overall complication rate was not significantly different between the two groups $(p=0.2575)$, whereas the postoperative pulmonary complication rate was significantly higher in the $\mathrm{C}$ group compared to the ER group ( $28.5 \%$ vs. $9.5 \%, p=0.0235)$. The group differences in anastomotic leakage were not statistically significant. Time to oral feeding was shorter in ER group than in $\mathrm{C}$ group (4.5 days vs. 9 days, $p<0.0001$ ). Time to flatus and defecation did not differ between the two groups. There were no significant differences in re-intubation and re-fasting between the two groups. Maximum dosage of EN in ER group was significantly fewer than in $\mathrm{C}$ group

Table 3 Demographic and clinical characteristics of all patients and propensity score-matched patients

\begin{tabular}{|c|c|c|c|c|c|c|}
\hline & \multicolumn{2}{|l|}{ All patient } & \multirow[t]{2}{*}{$p$ value } & \multicolumn{2}{|c|}{ Propensity score-matched patients } & \multirow[t]{2}{*}{$p$ value } \\
\hline & $\begin{array}{l}\text { Conventional } \\
\text { protocol }(n=73)\end{array}$ & $\begin{array}{l}\text { Enhanced recovery } \\
\text { protocol }(n=46)\end{array}$ & & C group $(n=42)$ & ER group $(n=42)$ & \\
\hline Sex, $n(\%)$ & & & 0.5391 & & & 0.3914 \\
\hline Male & $59(80.8 \%)$ & $35(76.1 \%)$ & & $36(85.7 \%)$ & $33(78.6 \%)$ & \\
\hline Female & $14(19.2 \%)$ & $11(23.9 \%)$ & & $6(14.3 \%)$ & $9(21.4 \%)$ & \\
\hline Age, year, mean \pm SD & $67.1 \pm 7.7$ & $67.2 \pm 8.6$ & 0.3437 & $68.3 \pm 5.8$ & $68.4 \pm 6.0$ & 0.9414 \\
\hline BMI, $\mathrm{kg} / \mathrm{m} 2$, mean $\pm \mathrm{SD}$ & $22.0 \pm 3.5$ & $21.6 \pm 3.2$ & 0.5134 & $22.1 \pm 3.6$ & $21.8 \pm 3.3$ & 0.6463 \\
\hline Serum albumin, $g / d L$, mean $\pm S D$ & $3.9 \pm 0.4$ & $4.0 \pm 0.4$ & 0.2694 & $3.9 \pm 0.4$ & $4.0 \pm 0.4$ & 0.2888 \\
\hline Cholinesterase, U/L, mean \pm SD & $290.3 \pm 70.9$ & $284.1 \pm 72.8$ & 0.6480 & $286.5 \pm 72.0$ & $277.1 \pm 66.3$ & 0.5352 \\
\hline Serum transthyretin, $\mathrm{mg} / \mathrm{dL}$, mean $\pm \mathrm{SD}$ & $26.3 \pm 5.4$ & $26.3 \pm 4.4$ & 0.9586 & $26.3 \pm 5.4$ & $26.4 \pm 4.4$ & 0.9298 \\
\hline ASA-PS & & & 0.2022 & & & 0.8413 \\
\hline 1 & $15(20.5 \%)$ & $7(15.2 \%)$ & & $8(19.0 \%)$ & $6(14.3 \%)$ & \\
\hline 2 & $52(71.2 \%)$ & $38(82.6 \%)$ & & $33(78.6 \%)$ & $35(83.3 \%)$ & \\
\hline 3 & $6(8.3 \%)$ & $1(2.4 \%)$ & & $1(2.4 \%)$ & $1(2.4 \%)$ & \\
\hline Histology & & & 0.2363 & & & 0.2950 \\
\hline Squamous cell carcinoma & $69(94.6 \%)$ & $43(93.5 \%)$ & & $41(97.6 \%)$ & $39(92.9 \%)$ & \\
\hline Adenocarcinoma & $2(2.7 \%)$ & $3(6.5 \%)$ & & $1(2.4 \%)$ & $3(7.1 \%)$ & \\
\hline Basaloid carcinoma & $2(2.7 \%)$ & & & & & \\
\hline Preoperative chemotherapy & & & 0.7186 & & & 0.8232 \\
\hline Yes & $42(57.5 \%)$ & $28(60.9 \%)$ & & $25(59.5 \%)$ & $26(61.9 \%)$ & \\
\hline No & $31(42.5 \%)$ & $18(39.1 \%)$ & & $17(40.5 \%)$ & $16(38.1 \%)$ & \\
\hline Clinical stage, $n(\%)$ & & & 0.9660 & & & 0.9699 \\
\hline $0 / I$ & $28(38.2 \%)$ & $15(32.6 \%)$ & & $17(40.5 \%)$ & $15(35.7 \%)$ & \\
\hline II & $25(34.3 \%)$ & $17(37.0 \%)$ & & $13(31.0 \%)$ & $15(35.7 \%)$ & \\
\hline III & $15(20.6 \%)$ & $11(23.9 \%)$ & & $9(21.4 \%)$ & $10(23.8 \%)$ & \\
\hline IVA & $5(6.9 \%)$ & $3(6.5 \%)$ & & $3(7.1 \%)$ & $2(4.8 \%)$ & \\
\hline
\end{tabular}

$S D$ standard deviation; $B M I$ body mass index; $A S A-P S$ american society of anesthesiologists physical status 
Table 4 Perioperative outcomes

\begin{tabular}{|c|c|c|c|c|c|c|}
\hline & \multicolumn{2}{|l|}{ All patient } & \multirow[t]{2}{*}{$p$ value } & \multicolumn{2}{|c|}{ Propensity score-matched patients } & \multirow{2}{*}{$p$ value } \\
\hline & $\begin{array}{l}\text { Conventional } \\
\text { protocol } \\
(n=73)\end{array}$ & $\begin{array}{l}\text { Enhanced } \\
\text { recovery protocol } \\
(n=46)\end{array}$ & & C group $(n=42)$ & ER group $(n=42)$ & \\
\hline \multicolumn{7}{|l|}{ Intraoperative outcome } \\
\hline Operative time, mean $\pm \mathrm{SD}$, minute & $548.9 \pm 68.7$ & $546.8 \pm 73.4$ & 0.8766 & $549.4 \pm 74.8$ & $550.4 \pm 71.3$ & 0.9537 \\
\hline Bleeding, mean $\pm \mathrm{SD}, \mathrm{ml}$ & $188.6 \pm 131.3$ & $172.1 \pm 146.9$ & 0.5113 & $188.6 \pm 131.3$ & $177.6 \pm 152.3$ & 0.7239 \\
\hline Fluid balance, mean $\pm \mathrm{SD}, \mathrm{ml}$ & $4034.4 \pm 989.5$ & $3916.7 \pm 1024.4$ & 0.5342 & $4135.1 \pm 1061.4$ & $3922.2 \pm 1009.2$ & 0.3489 \\
\hline Use of catecholamine, $n(\%)$ & $70(95.9 \%)$ & $41(89.1 \%)$ & 0.1583 & $41(97.6 \%)$ & $38(90.5 \%)$ & 0.1531 \\
\hline \multicolumn{7}{|l|}{ Lymphadenectomy } \\
\hline 3-fields & $64(87.6 \%)$ & $34(73.9 \%)$ & 0.0584 & $37(88.1 \%)$ & $32(76.2 \%)$ & 0.1510 \\
\hline 2-fields & $9(12.3 \%)$ & $12(26.1 \%)$ & & $5(11.9 \%)$ & $10(23.8 \%)$ & \\
\hline \multicolumn{7}{|l|}{ Postoperative outcome } \\
\hline Time to extubation, median, day, range & $1(0-55)$ & $0(0-1)$ & $<0.0001 *$ & $1(0-1)$ & $0(0-1)$ & $<0.0001 *$ \\
\hline Use of catecholamine, $n(\%)$ & $68(93.2 \%)$ & $10(21.7 \%)$ & $<0.0001 *$ & $39(92.8 \%)$ & $7(16.7 \%)$ & $<0.0001 *$ \\
\hline Length of ICU stay, median, day, range & $2(2-94)$ & $2(0-6)$ & 0.0628 & $2(2-94)$ & $2(2-3)$ & 0.2192 \\
\hline Time to ambulation, median, day, range & $3(3-24)$ & $2(2-11)$ & $<0.0001 *$ & $3(3-22)$ & $2(2-11)$ & $<0.0001 *$ \\
\hline $\begin{array}{l}\text { Vocal cord palsy (swallowing with VE } \\
\text { CD >I), } n(\%)\end{array}$ & $8(11.0 \%)$ & $8(17.4 \%)$ & 0.3219 & $6(14.3 \%)$ & $8(19.1 \%)$ & 0.5576 \\
\hline Time to oral feeding, median, day, range & $9(7-46)$ & $4.5(4-44)$ & $<0.0001 *$ & $9(7-46)$ & $4.5(4-37)$ & $<0.0001^{*}$ \\
\hline Time to flatus, median, day, range & $2(1-5)$ & $2(1-5)$ & 0.7434 & $3(1-5)$ & $2(1-5)$ & 0.6617 \\
\hline Time to defecation, median, day, range & $3(2-6)$ & $3(2-6)$ & 0.5744 & $3(2-6)$ & $3(2-6)$ & 0.5470 \\
\hline Overall morbidity (CD > II), $n(\%)$ & $37(50.0 \%)$ & $14(30.4 \%)$ & $0.0283^{*}$ & $18(42.9 \%)$ & $13(30.9 \%)$ & 0.2575 \\
\hline Pulmonary complications, $n(\%)$ & $20(27.4 \%)$ & $4(8.7 \%)$ & $0.0094 *$ & $12(28.5 \%)$ & $4(9.5 \%)$ & $0.0235^{*}$ \\
\hline Anastomotic leakage, $n(\%)$ & $6(8.2 \%)$ & $6(13.0 \%)$ & 0.4003 & $3(7.1 \%)$ & $5(11.9 \%)$ & 0.4551 \\
\hline Chylothorax, $n(\%)$ & $3(4.1 \%)$ & $0(0 \%)$ & 0.0843 & $2(4.8 \%)$ & $0(0 \%)$ & 0.0930 \\
\hline \multicolumn{7}{|l|}{$\mathrm{CRP}$, mean $\pm \mathrm{SD}, \mathrm{mg} / \mathrm{dL}$} \\
\hline POD 1 & $7.4 \pm 1.9$ & $7.6 \pm 2.9$ & 0.2622 & $7.0 \pm 1.8$ & $7.7 \pm 2.7$ & 0.2148 \\
\hline POD 3 & $14.2 \pm 8.4$ & $13.6 \pm 7.8$ & 0.9108 & $13.8 \pm 8.4$ & $13.9 \pm 7.5$ & 0.9388 \\
\hline POD 7 & $6.6 \pm 5.6$ & $5.2 \pm 5.5$ & 0.4996 & $6.1 \pm 5.2$ & $5.1 \pm 5.7$ & 0.4780 \\
\hline POD 10 & $3.2 \pm 3.5$ & $2.8 \pm 3.4$ & 0.5575 & $3.2 \pm 3.3$ & $2.7 \pm 3.6$ & 0.5849 \\
\hline $\begin{array}{l}\text { Maximum dosage of } \mathrm{EN} \text {, mean } \pm \mathrm{SD}, \mathrm{ml} / \\
\text { day }\end{array}$ & $1380.8 \pm 268.9$ & $1053.2 \pm 186.7$ & $<0.0001^{*}$ & $1394.3 \pm 269.0$ & $1050.0 \pm 190.0$ & $<0.0001^{*}$ \\
\hline $\begin{array}{l}\text { Maximum calorie intake, mean } \pm \text { SD, } \\
\text { kcal/day }\end{array}$ & $1489.7 \pm 203.8$ & $1455.9 \pm 199.0$ & 0.3750 & $1483.3 \pm 214.1$ & $1470.2 \pm 193.8$ & 0.7697 \\
\hline $\begin{array}{l}\text { Abdominal dysfunction related to EN, } n \\
(\%)\end{array}$ & $28(38.4 \%)$ & $9(19.6 \%)$ & $0.0485^{*}$ & $18(42.9 \%)$ & $7(16.7 \%)$ & $0.0078^{*}$ \\
\hline Re-intubation, $n(\%)$ & $5(6.8 \%)$ & $1(2.2 \%)$ & 0.2292 & $3(7.1 \%)$ & $1(2.4 \%)$ & 0.2950 \\
\hline Re-fasting, $n(\%)$ & $9(12.5 \%)$ & $8(17.4 \%)$ & 0.4642 & $8(16.7 \%)$ & $6(14.2 \%)$ & 0.7628 \\
\hline $\begin{array}{l}\text { Length of hospital stay, median, day, } \\
\text { range }\end{array}$ & $23(14-317)$ & $17.5(12-72)$ & $0.0007 *$ & $23(16-317)$ & $17.5(12-63)$ & $0.0034^{*}$ \\
\hline In-hospital mortality & N/A & N/A & & N/A & N/A & \\
\hline
\end{tabular}

$S D$ standard deviation; $I C U$ intensive care unit; $E N$ enteral nutrition; $C D$ Clavien Dindo classification; $C R P C$-reactive protein; $P O D$ postoperative day; N/A not applicable

$(1050.0 \pm 190.0 \mathrm{kcal}$ vs. $1394.3 \pm 269.0 \mathrm{kcal}, p<0.0001)$, while no differences in maximum total calorie intake were observed between both groups. The $\mathrm{C}$ group had higher ENrelated abdominal discomfort compared to the ER group ( $42.9 \%$ vs. $16.7 \%, p=0.0078$ ). The length of hospital stay in the ER group was significantly shorter than in the $\mathrm{C}$ group
(17.5 days vs. 23 days, $p=0.0034$ ). In-hospital mortality was not observed in the two groups.

Figure 2 shows the perioperative nutritional physical examination maintenance rate before and after matching. The median loss of body weight ( $6.3 \%$ vs. $7.7 \%, p=0.0065)$ and BMI $(5.6 \%$ vs. $8.1 \%, p=0.0017)$ were significantly 


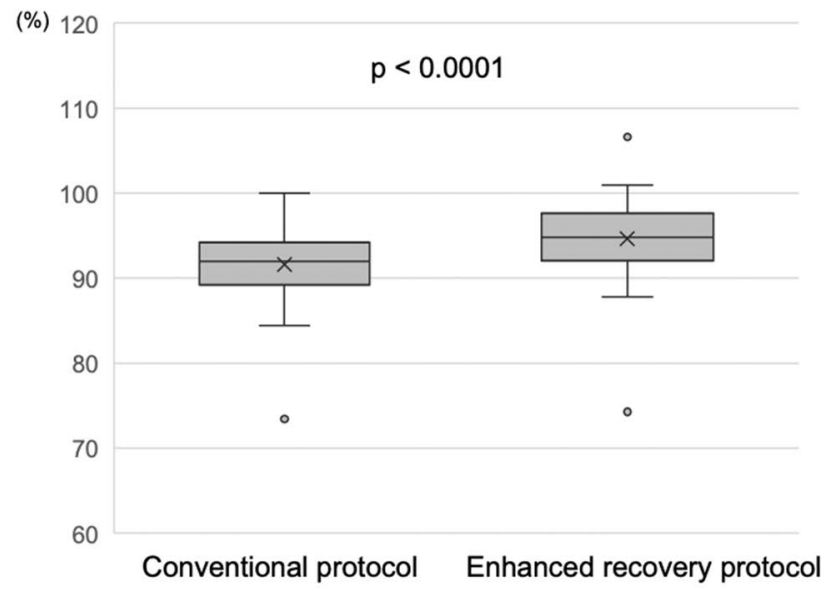

(a) Before matching, BW

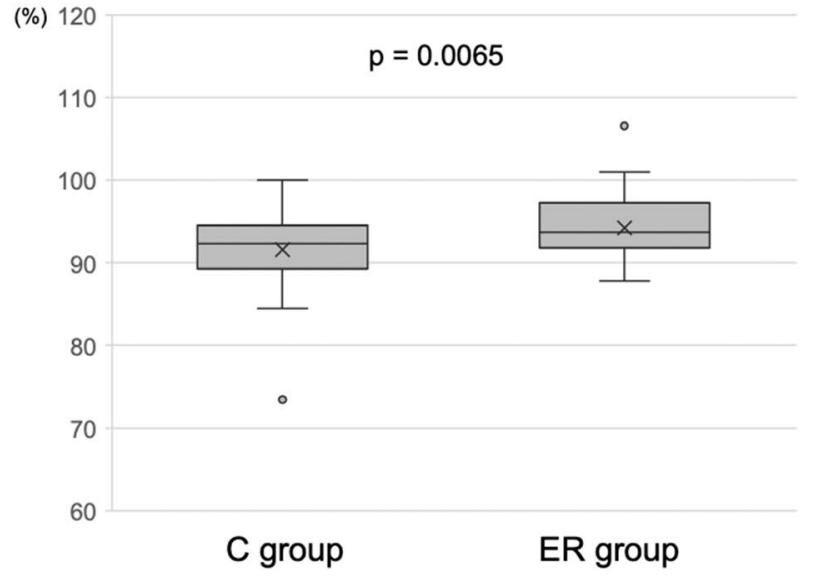

(c) After matching, BW

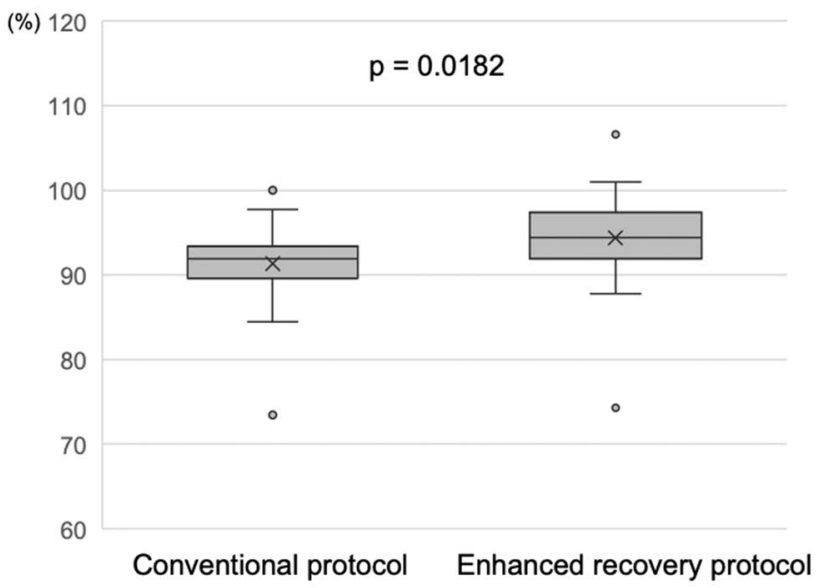

(b) Before matching, BMI

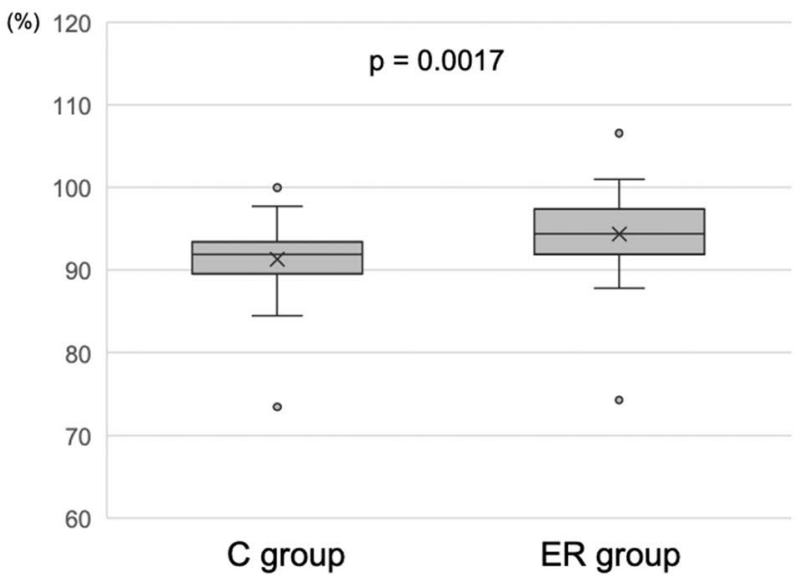

(D) After matching, BMI

Fig. 2 Difference in postoperative nutritional status for a BW, b BMI before matching. Difference in postoperative nutritional status for $\mathbf{c}$ BW, d BMI after matching. $B W$ body weight, $B M I$ body mass index

lower in the ER group than in the $\mathrm{C}$ group one month postoperatively.

Figure 3 shows perioperative nutritional biochemistry data one month after surgery before and after matching. There was no significant difference between both groups for Alb (88.5\% vs. 89.4\%), ChE (78.2\% vs. 81.8\%), and TTR $(82.4 \%$ vs. $85.5 \%)$.

\section{Discussion}

This study demonstrates the safety and feasibility of an enhanced recovery protocol in patients who underwent $\mathrm{MIMcE}$ for esophageal carcinoma. Implementation of our enhanced recovery protocol includes early extubation and successful oral intake without increasing respiratory and anastomotic complications.

ERAS was introduced as a perioperative management protocol to maintain physiological function and early recovery after surgery, and has now been popularized for some types of major gastrointestinal surgery, including esophagectomy [7-9]. However, there are few reports on ERAS, especially after MIMcE.

Lassen et al. reported that oral resumption on the first day after major upper gastrointestinal (UGI) surgery does not increase postoperative complications as compared to conventional care with nil-by-mouth $(28 \%$ vs. $36 \%, p=0.26)$ [10]; however, although ERAS has been applied to common UGI surgeries, only $2.7 \%$ of patients undergoing transthoracic esophagectomy in the above study were included. ERAS protocols, including the timing of oral intake, remain controversial. Weijs et al. reported that initiation of oral intake on POD 1 after esophagectomy did not increase pneumonia and the severity of anastomotic leakage [12]. However, their main procedure was Ivor Lewis, which is different from our procedure of McKeown esophagectomy. McKeown esophagectomy has additional risks for laryngeal elevation disorder and recurrent nerve palsy compared to 


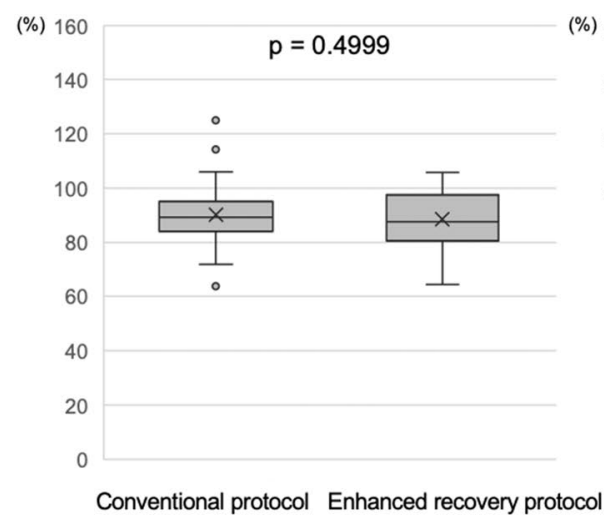

(a) Before matching, Alb

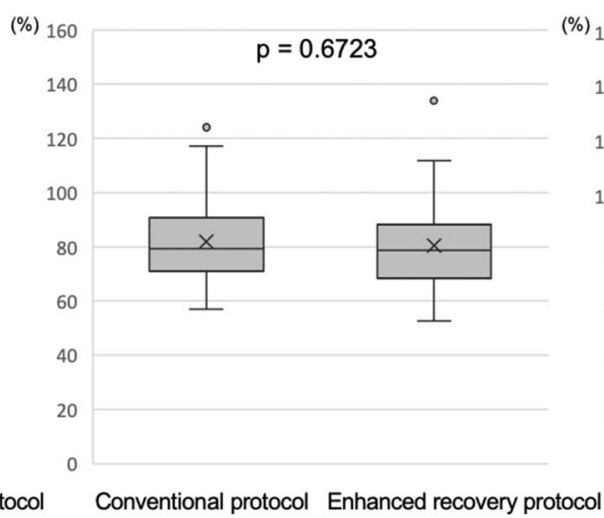

(b) Before matching, ChE

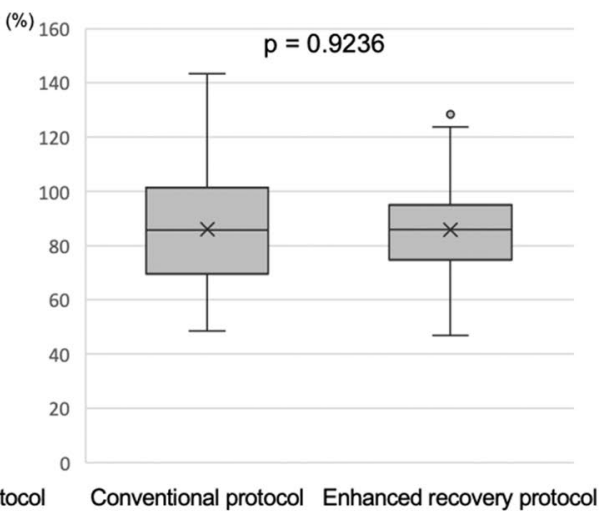

(c) Before matching, TTR

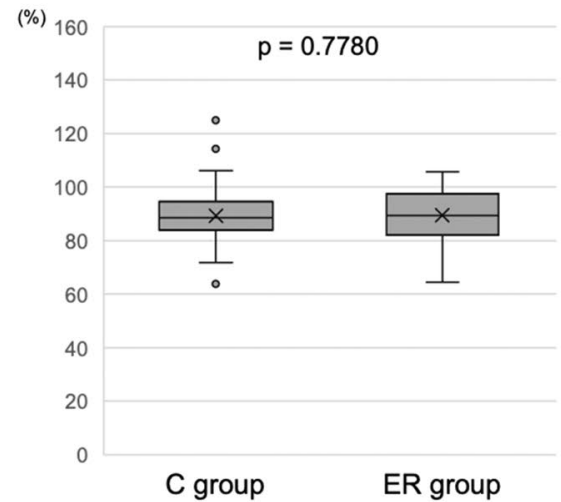

(d) After matching, Alb

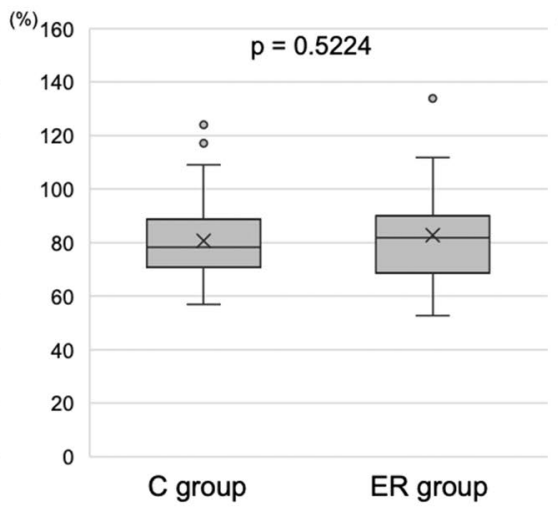

(e) After matching, ChE

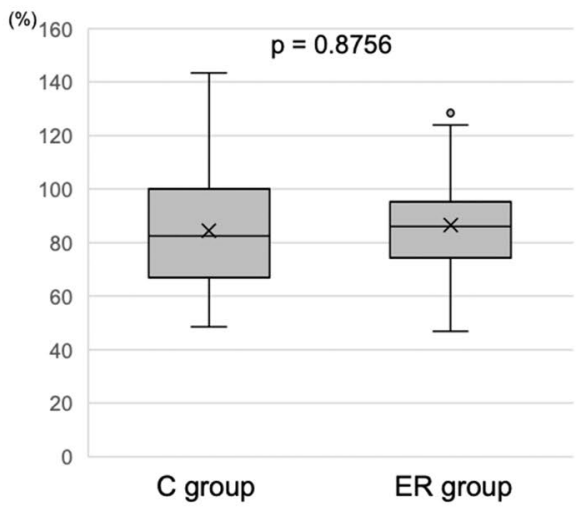

(f) After matching, TTR

Fig. 3 Maintenance of nutritional biochemistry data for $\mathbf{a} \mathrm{Alb}, \mathbf{b} \mathrm{ChE}$, and $\mathbf{c}$ TTR before matching. Maintenance of nutritional biochemistry data for $\mathbf{d ~ A l b , ~ e ~ C h E , ~ a n d ~} \mathbf{f}$ TTR before matching. Alb serum albumin, ChE cholinesterase, TTR serum transthyretin

Ivor Lewis [13]. Sun et al. reported early oral feeding on POD 1 for patients who underwent esophagectomy, including 3.2\% of 3-field lymphadenectomy, was similar to late oral feeding on POD 7 with regard to postoperative pulmonary complications $(22.1 \%$ vs. $28.6 \%, 95 \%$ confidence interval (CI): $-16.7-3.7 \%)$ and anastomotic leak (3.6\% vs. $4.3 \%$, 95\% CI: $-5.3-3.9 \%)$. In addition, they had a quicker recovery of bowel function and improved short-term quality of life [14]. Most patients in this study required cervical lymphadenectomy due to oncological background. Overall complications and anastomotic leakage between the two groups were similar to previous outcomes, which support postoperative early recovery $[15,16]$.

In the conventional protocol, we managed the patient on ventilation until POD 1 in the ICU to prevent aspiration, secure the airway, and control pain. However, ventilator management (VM) carries the risk of ventilator-associated pneumonia, lung injury, and sedation-related complications. Lanuti et al. advocate in their report that most patients who underwent esophagectomy were safely extubated immediately with appropriate fluid balance, limited blood loss, and adequate anesthetic and analgesic techniques [17]. Several reports have shown that immediate extubation (IE) after McKeown esophagectomy is feasible and safe. Toh et al. demonstrated that IE tended to reduce the incidence of pulmonary complications compared with VM (12\% vs. $24 \%$, NS) [18]. Imai et al. reported that the rate of ambulation on POD 1 was significantly higher in the IE group than in the VM group (50 vs. $19 \%, p=0.003$ ) which resulted in a shorter ICU stay [3 (2.75-3) days vs. 3 (3-4) days, $p=0.01$, without increasing postoperative complications [19]. Time to ambulation was shorter in the ER group than in the $\mathrm{C}$ group. Although morbidity did not differ between the groups, pulmonary complications were significantly lower in the ER group than in the $\mathrm{C}$ group. This is because early mobilization is an indispensable part of ERAS protocol. Prolonged bed rest in the postoperative period causes muscle loss, atelectasis, pulmonary complications, and venous thromboembolism [20]. For the prevention of these problems, the ER group received adequate early physical rehabilitation after esophagectomy, preferably in the ICU. Based on these results, early extubation and active rehabilitation 
associated with an enhanced protocol should be recommended for favorable postoperative respiratory management after MIMcE.

Traditionally, NG tube after esophagectomy was considered useful for reflux or decompression of anastomosis compared to other gastrointestinal surgery. In a systematic review and meta-analysis, there was no difference in anastomotic leakage (risk ratio (RR):0.85, 95\% CI: 0.46-1.54), postoperative pneumonia (RR:1.27, 95\% CI: $0.70-2.29$ ) and mortality (RR:0.71, 95\% CI: 0.30-1.69) between immediate or early and late removal NG tube following esophagectomy [21]. Contrastingly, Zhang et al. reported NG tube placement does not decrease the risk of anastomotic leak and postoperative pneumonia in esophagectomy patients; the patients without NG tube had faster bowel function recovery and shorter hospital length of stay [22]. Our results were aligned with this hence we conclude that our ERAS protocol with the complete omission of NG tube is feasible.

Feeding jejunostomy or gastrostomy for feeding is generally performed during esophagectomy. The usefulness of early postoperative enteral feeding has been demonstrated in several reports. Early postoperative EN may be able to improve the immune system and suppress excessive inflammatory responses in patients who have undergone gastrointestinal surgery [23]. Aiko at el. have shown that EN following esophagectomy was associated with fewer postoperative complications, shorter hospital stays, and a lower level of C-reactive protein (CRP) than total parental nutrition [24]. In this study, early postoperative EN was applied in both protocols. The trend of postoperative CRP in the two groups were not significantly different, thus, an addition of early oral intake to early postoperative EN may not affect the worsening of inflammation after MIMcE.

Implementation of the ERAS protocol after esophagectomy was successful with respect to complications, postoperative hospital stay, and costs [8, 11-22, 25]. In addition, postoperative EN after thoracoscopic esophagectomy was more favorable than total parental nutrition for bodyweight loss $(-2.94 \pm 3.19 \%$ vs. $5.05 \pm 3.65 \%, \mathrm{P}=0.020)$ [26]. In our study, we also focused on perioperative changes in a physical examination and biochemical nutritional index. Currently, there is evidence to support initiating enteral nutrition early and reaching full-calorie requirement target at 3-6 days following esophagectomy, whereas, there is no consensus on the flow rate of the nutrient with jejunostomy after esophagectomy [27]. Desistance of postoperative body weight and BMI were better in the ER group than in the $\mathrm{C}$ group. The flow rate of $\mathrm{EN}$ in $\mathrm{C}$ group seemed to exceed the intestinal tolerance to volume loading, therefore, abdominal dysfunction-related EN were more frequently observed in $\mathrm{C}$ group. Consequently, these symptoms may be related to the difference in weight loss between the two groups. It is considered that early oral feeding for target calorie intake in the ER group reduced abdominal symptoms and may have affected the $1.4 \%$ difference in BW maintenance between both groups. Notably, body weight loss and subnutrition after esophagectomy are known prognostic factors for esophageal cancer. Furthermore, one previous study demonstrated that severe transthyretin, which decreased after esophagectomy, was shown to be a risk factor for anastomotic leak after MIE [28]. Thus, an enhanced recovery protocol can also be beneficial for the improvement of prognosis for patients with esophageal cancer [29]. On the other hand, the change in biochemical nutritional data was not significant in either group in this study. $\mathrm{Li}$ et al. reported that perioperative changes in albumin and transthyretin, as well as morbidity, were not significantly different between the early oral intake and one-week tube feeding groups [30]. Given the above findings, biochemical nutritional markers may not change as compared to the change in body weight or BMI.

In general, ASA-PS 3 is associated with negative postoperative outcomes. There was no statistic dispersion in ASAPS before matching. Five patients with ASA-PS 3 had been excluded after propensity score matching; the confounding factor was adjusted for the patient background associated with postoperative outcomes. Therefore, a more appropriate comparison could be achieved after matching.

This study has several limitations. First, this was a single-center study with a small number of patients. A multicenter collaborative study with a large cohort might provide more definitive results. Second, nutrition management after discharge were not been matched. Third, BW, BMI, and biochemistry data may be inadequate indicators of nutrition assessment.

In conclusion, this study shows that an enhanced recovery protocol can be applied safely to patients receiving MIMcE for esophageal carcinoma without worsening postoperative nutritional status as well as minimizing complications.

Acknowledgments We thank all the members of the nutritional support team in Jikei University Hospital. The authors also thank Editage (www. editage.com) for English language editing.

Author contributions Design of the study: Yuichiro Tanishima; Analysis of data: Yuichiro Tanishima; Drafting of the article: Yuichiro Tanishima; Revision of the article: KN, TI; Collection of data: Yuichiro Tanishima, MY, AM, Yujiro Tanaka, YI, KT, FY, NM; Final approval of the article: TI.

\section{Compliance with ethical standards}

Ethical statement All procedures followed were in accordance with the ethical standards of the responsible committee on human experimentation (institutional and national).

Conflict of interest All authors declare that they have no conflict of interest. 
Informed consent The Helsinki Declaration of 1964 and later versions. Informed consent was obtained from all patients for being included in the study.

\section{References}

1. Bollschweiler E, Plum P, Monig SP, et al. Current and future treatment options for esophageal cancer in the elderly. Expert Opin Pharmacother. 2017;18:1001-10.

2. Tachimori Y, Ozawa S, Numasaki H, et al. Comprehensive registry of esophageal cancer in Japan, 2012. Esophagus. 2019;16:221-45.

3. Takeuchi H, Miyata H, Ozawa S, et al. Comparison of short-term outcomes between open and minimally invasive esophagectomy for esophageal cancer using a nationwide database in Japan. Ann Surg Oncol. 2017;24:1821-7.

4. Kakeji Y, Takahashi A, Hasegawa H, et al. Surgical outcomes in gastroenterological surgery in Japan: report of the National Clinical Database 2011-2018. Ann Gastroenterol Surg. 2020;4:250-74.

5. Gottlieb-Vedi E, Kauppila JH, Malietzis G, et al. Long-term survival in esophageal cancer after minimally invasive compared to open esophagectomy: a systematic review and meta-analysis. Ann Surg. 2019;270:1005-17.

6. Kammili A, Cools-Lartigue J, Mulder D, et al. Transition from open to minimally invasive en bloc esophagectomy can be achieved without compromising surgical quality. Surg Endosc. 2020. https://doi.org/10.1007/s00464-020-07696-0.

7. Fearon KC, Ljungqvist O, Von Meyenfeldt M, et al. Enhanced recovery after surgery: a consensus review of clinical care for patients undergoing colonic resection. Clin Nutr. 2005;24:466-77.

8. Giacopuzzi S, Weindelmayer J, Treppiedi E, et al. Enhanced recovery after surgery protocol in patients undergoing esophagectomy for cancer: a single center experience. Dis Esophagus. 2017;30:1-6. https://doi.org/10.1093/dote/dow024.

9. Visioni A, Shah R, Gabriel E, et al. Enhanced recovery after surgery for noncolorectal surgery? A systematic review and metaanalysis of major abdominal surgery. Ann Surg. 2018;267:57-65.

10. The Japan Esophageal Society. Guidelines for diagnosis and treatment of carcinoma of the esophagus 2017. Tokyo: Kanehara Co Ltd; 2017. ((in Japanese)).

11. Lassen K, Kjæve J, Fetveit T, et al. Allowing normal food at will after major upper gastrointestinal surgery does not increase morbidity: a randomized multicenter trial. Ann Surg. 2008;247:721-9.

12. Weijs TJ, Berkelmans GH, Nieuwenhuijzen GA, et al. Immediate postoperative oral nutrition following esophagectomy: a multicenter clinical trial. Ann Thorac Surg. 2016;102:1141-8.

13. Jezerskyte E, Saadeh LM, Hagens ERC, et al. Long-term healthrelated quality of life after McKeown and Ivor Lewis esophagectomy for esophageal carcinoma. Dis Esophagus. 2020. https://doi. org/10.1093/dote/doaa022.

14. Sun HB, Li Y, Liu XB, et al. Early oral feeding following McKeown minimally invasive esophagectomy: an openlabel, randomized, controlled, noninferiority trial. Ann Surg. 2018;267:435-42.

15. Ashok A, Niyogi D, Ranganathan $\mathrm{P}$, et al. The enhanced recovery after surgery (ERAS) protocol to promote recovery following esophageal cancer resection. Surg Today. 2020;50:323-34.
16. Triantafyllou T, Olson MT, Theodorou D, et al. Enhanced recovery pathways vs standard care pathways in esophageal cancer surgery: systematic review and meta-analysis. Esophagus. 2020;17:100-12.

17. Lanuti M, de Delva PE, Maher A, et al. Feasibility and outcomes of an early extubation policy after esophagectomy. Ann Thorac Surg. 2006;82:2037-41.

18. Toh Y, Oki E, Minami K, et al. Evaluation of the feasibility and safety of immediate extubation after esophagectomy with extended radical three-field lymph node dissection for thoracic esophageal cancers. Esophagus. 2009;6:167-72.

19. Imai $\mathrm{T}, \mathrm{Abe} \mathrm{T}$, Uemura $\mathrm{N}$, et al. Immediate extubation after esophagectomy with three-field lymphadenectomy enables early ambulation in patients with thoracic esophageal cancer. Esophagus. 2018;15:165-72.

20. Low DE, Allum W, De Manzoni G, et al. Guidelines for perioperative care in esophagectomy: enhanced recovery after surgery $\left(\right.$ ERAS $\left.^{\circledR}\right)$ society recommendations. World J Surg. 2019;43:299-330.

21. Weijs TJ, Kumagai K, Berkelmans GH, et al. Nasogastric decompression following esophagectomy: a systematic literature review and meta-analysis. Dis Esophagus. 2017;30:1-8.

22. Zhang R, Zhang L. Feasibility of complete nasogastric tube omission in esophagectomy patients. J Thorac Dis. 2019;11:S819-23.

23. Lobo DN, Williams RN, Welch NT, et al. Early postoperative jejunostomy feeding with an immune-modulating diet in patients undergoing resectional surgery for upper gastrointestinal cancer: a prospective, randomized, controlled, double-blind study. Clin Nutr. 2006;25:716-26.

24. Aiko S, Yoshizumi Y, Sugiura Y, et al. Beneficial effects of immediate enteral nutrition after esophageal cancer surgery. Surg Today. 2001;31:971-8.

25. Weindelmayer J, Verlato G, Alberti L, et al. Enhanced recovery protocol in esophagectomy, is it really worth it? A cost analysis related to team experience and protocol compliance. Dis Esophagus. 2019;32: doy114.

26. Takesue T, Takeuchi $\mathrm{H}$, Ogura $\mathrm{M}$, et al. A prospective randomized trial of enteral nutrition after thoracoscopic esophagectomy for esophageal cancer. Ann Surg Oncol. 2015;22:S802-9.

27. Weimann A, Braga M, Carli F, et al. ESPEN guideline: clinical nutrition in surgery. Clin Nutr. 2017;36:623-50.

28. Wang YJ, Liu XH, Mei LY, et al. Do alterations in plasma albumin and prealbumin after minimally invasive esophagectomy for squamous cell carcinoma influence the incidence of cervical anastomotic leak? Surg Endosc. 2016;30:3943-9.

29. Kubo Y, Miyata H, Sugimura K, et al. Prognostic implication of postoperative weight loss after esophagectomy for esophageal squamous cell cancer. Ann Surg Oncol. 2020. https://doi. org/10.1245/s10434-020-08762-6.

30. Li Y, Liu Z, Liu G, et al. Impact on short-term complications of early oral feeding in patients with esophageal cancer after esophagectomy. Nutr Cancer. 2020. https://doi.org/10.1080/01635 581.2020 .1769690 .

Publisher's Note Springer Nature remains neutral with regard to jurisdictional claims in published maps and institutional affiliations. 